\title{
Successful Management of Prosthetic Valve Brucella Endocarditis with Antibiotherapy Alone
}

\author{
José Pedro Fonseca ${ }^{1}$, Telma Pereiro ${ }^{1}$, Diana Pinho dos Santos ${ }^{1}$, José Miguel Correia $^{2}$, Joana Capelo ${ }^{1}$, Adelino Carragoso ${ }^{1}$ \\ ${ }^{1}$ Internal Medicine Service, Centro Hospitalar Tondela-Viseu, E.P.E. - Viseu, Portugal \\ ${ }^{2}$ Cardiology Service, Centro Hospitalar Tondela-Viseu, E.P.E. - Viseu, Portugal
}

\section{Doi: 10.12890/2018_000808 - European Journal of Case Reports in Internal Medicine - ๑ EFIM 2018}

Received: 08/12/2017

Accepted: $18 / 12 / 2017$

Published: $30 / 01 / 2018$

How to cite this article: Fonseca JP, Pereiro T, Pinho dos Santos D, Correia JM, Capelo J, Carragoso A. Successful management of prosthetic valve Brucella endocarditis with antibiotherapy alone. EJCRIM 2018;5: doi:10.12890/2018_000808.

Conflicts of Interests: The Authors declare that there are no competing interests.

This article is licensed under a Commons Attribution Non-Commercial 4.0 License

\section{ABSTRACT}

Objectives: To report a case of mechanical aortic prosthesis Brucella endocarditis successfully treated with antibiotics alone.

Materials and methods: We describe a clinical case and present a review of the literature.

Results: A 60-year-old female farmer with a mechanical aortic prosthetic valve presented with low back pain and fever. She was diagnosed with prosthetic valve Brucella mellitensis endocarditis and was cured with antibiotic therapy alone. Few cases of successfully treated prosthetic valve Brucella endocarditis without surgery have been reported.

Conclusion: Prosthetic valve Brucella endocarditis usually requires surgical valve replacement. However, selected patients may be successfully treated with antibiotic therapy alone.

\section{LEARNING POINTS}

- Brucella endocarditis is responsible for most fatal cases of brucellosis.

- Brucellosis relapse after treatment in patients with a cardiac valve prosthesis should arouse suspicion for endocarditis.

- Long-term medical treatment alone can be successful in selected patients with Brucella endocarditis, even in those with prosthetic valve endocarditis..

\section{KEYWORDS}

Brucellosis, Brucella endocarditis, prosthetic cardiac valve Brucella endocarditis, Brucella, Brucella mellitensis

\section{INTRODUCTION}

Brucellosis is a systemic infectious disease that can affect any organ or tissue. The infection may present with or be complicated by specific organ involvement (focal brucellosis). It may have chronic courses, therapeutic failures and relapses ${ }^{[1]}$.

Brucellosis is a zoonosis with a worldwide distribution, being common in Mediterranean countries. It is usually acquired through ingestion of infected dairy products, mainly unpasteurized goat's milk and cheese, but may also be transmitted by direct animal contact or inhalation of infectious aerosols ${ }^{[2]}$.

The case fatality rate in brucellosis is low (less than 1\%), with Brucella endocarditis (BE), a rare focal complication, responsible for $80 \%$ of fatal cases $^{[3]}$. 
Brucella endocarditis is a slowly destructive process, with a high tendency to tissue ulceration, abscess formation, and the development of large vegetations carrying a significant risk of embolization and difficulty in eradicating the infection with medical treatment alone ${ }^{[4]}$.

Some patients with BE have been cured by medical treatment alone, and rare cases of prosthetic valve BE cured with medical treatment have been reported ${ }^{[5-8]}$. In the present paper, we report the case of a patient with a mechanical aortic valve prosthesis BE that was successfully treated with long-term antibiotic therapy only.

\section{CASE REPORT}

A 60-year-old female farmer was hospitalized in March 2013 with a 3-month history of low back pain and with fever for the past week. She also reported weight loss and asthenia. Her past medical history was relevant for severe aortic stenosis with aortic valve replacement with a mechanical prosthetic valve (Medtronic Hall size 22) in 2008 and brucellosis diagnosed in February 2012 and treated by her primary care physician. She referred consumption of unpasteurized goat's milk and cheese prior to the diagnosis of brucellosis. She was medicated with warfarin.

On admission she was afebrile, haemodynamically stable with a blood pressure of $110 / 70 \mathrm{mmHg}$, heart rate of 90 beats/min, and oxygen saturation of $95 \%$. An aortic systolic murmur (grade II) was audible. There were no signs of heart failure. The liver and spleen were not enlarged on physical examination and no haemorrhages or petechia were found. She had limitation in lumbar movements with preserved strength and symmetric reflexes in the lower limbs.

Laboratory studies revealed a mild leukopenia with a total white cell count of 3,600/mm3 (66.7\% neutrophils, $23.6 \%$ lymphocytes, $9.1 \%$ monocytes), a slightly decreased number of platelets (142,000 platelets/ $\mu$ l) and a haemoglobin level of $11.8 \mathrm{~g} / \mathrm{dl}$. C-reactive protein was mildly elevated $(4.60 \mathrm{mg} / \mathrm{dl})$. Liver enzymes and renal function were normal. The serum (tube) agglutination test against Brucella species (Wright test) showed a positive titre of 1:320. Chest x-ray and ECG were normal.

A lumbar spine CT scan revealed only degenerative alterations. A transthoracic echocardiography (TTE) showed no signs of perivalvular complications or intracardiac masses.

Gram-negative coccobacilli were isolated from the initial blood cultures 4 days after incubation. Bone scintigraphy (MRI was not available) showed a focal increased uptake in the third lumbar vertebra (Fig. 1). The diagnosis of Brucella spondylodiscitis/spondylitis was considered, and the patient was given triple combination therapy consisting of oral doxycycline $100 \mathrm{mg}$ twice a day, oral rifampicin $600 \mathrm{mg}$ once daily and intravenous gentamycin ( $5 \mathrm{mg} / \mathrm{kg} / \mathrm{day})$.

Brucella was isolated from the three haemocultures drawn at admission after 8 days of incubation and Brucella mellitensis was later identified. Transoesophageal echocardiography (TEE) demonstrated several filiform vegetations attached to the aortic prosthetic aortic valve annulus with no signs of abscess or perivalvular leak (Fig. 2). The diagnosis of BE was made.

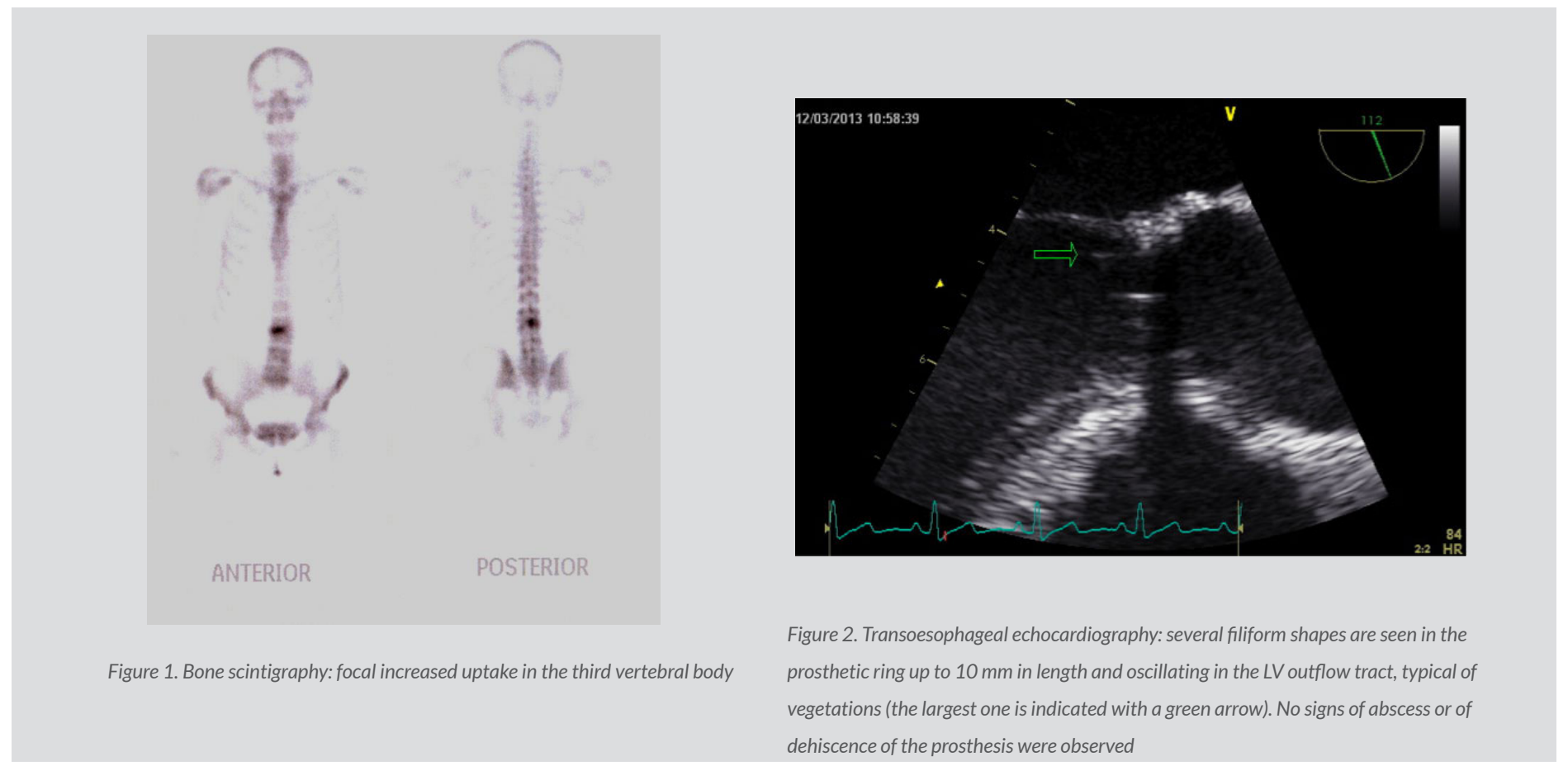


After the beginning of treatment, the patient remained afebrile and haemodynamically stable, with improvement of the complaints. A cardiothoracic surgery department was contacted to evaluate the need for valvular replacement. They decided not to intervene as there were no signs of heart failure, valvular malfunction or perivalvular complications at the time.

Gentamicin was discontinued after 3 weeks, and the patient was discharged with doxycycline 100 mg twice a day and oral rifampicin 600 mg once daily.

The patient was followed up at regular intervals for the next 12 months. Serial TTE demonstrated shrinkage of the vegetation. After 9 months, the Wright test was negative, and the antibiotics were stopped. Three months after the end of treatment the patient remained asymptomatic and with a negative Wright test.

Two years after the end of antibiotic therapy, the patient had no signs of relapse, the Wright test was still negative, and no signs of vegetations were visible on TEE.

\section{DISCUSSION}

Focal complications are found in one-third of all brucellosis patients, either initially or during the disease course ${ }^{[3]}$. Osteoarticular manifestations (sacroiliitis, spondylitis, peripheral arthritis, and osteomyelitis) account for over half of focal complications ${ }^{[1]}$. In Brucella spondylitis, the lumbar region is involved in $78 \%$ of cases $^{[9]}$.

Although BE as a focal complication of human brucellosis is reported in only $0.3-0.7 \%$ of cases, it has a high fatality rate (80\%) and is the most common cause of death in this disease ${ }^{[6]}$.

Approximately one-half of patients with BE have underlying valvular damage $\mathrm{e}^{[3]}$. Echocardiographic studies are mandatory in brucellosis patients with a history of valvular heart disease ${ }^{[3]}$.

Our patient had been treated for brucellosis diagnosed 1 year before the current admission and no echocardiographic study was performed. A cardiac focalization possibly acted as a source of perpetuation of infection causing spondylitis. The recurrence of bacteraemia after adequate antibiotic treatment of brucellosis in patients with prosthetic valves should always arouse suspicion of endocarditis ${ }^{[3]}$.

Because brucellosis is characterized by protean clinical manifestations, the diagnosis cannot be made solely on clinical grounds and it is essential to perform bacteriological and serological testing. A definitive diagnosis of human brucellosis requires isolation of the aetiological agent from blood, bone marrow or other tissues and body fluids ${ }^{[2]}$. Since culture techniques are time consuming, hazardous and not sensitive, most physicians rely on indirect proof of Brucella infection based on high or rising titres of specific antibodies ${ }^{[2]}$. The serum agglutination test (SAT) is generally accepted as the reference method in the serological diagnosis of human brucellosis. SAT titres $\geq 1: 160$ are generally considered consistent with active brucellosis in patients with a compatible clinical course and with a history of potential exposure, but in endemic areas titres $\geq 1: 320$ may be more specific ${ }^{[2]}$. Although the usefulness of serology in treatment follow-up is barely proven, definite cure of a patient correlates well with lower SAT titres ${ }^{[2]}$.

Confirmation of endocarditis is required through echocardiography. TEE may be very helpful in establishing the diagnosis as it has greater accuracy than TTE and provides more detailed information on valvular destruction, annular or myocardial abscesses, and the formation of aneurysms ${ }^{[3]}$. In this case, TEE was essential for diagnosis as it showed vegetations that were overlooked by TTE.

The propensity of Brucella to cause endocarditis associated with cardiac abscesses, large vegetations that frequently embolize resulting in infection and infarction in downstream organs, as well as the need for several antibiotics for minimum control of infection, has made surgery a mainstay of treatment compared with endocarditis caused by other organisms ${ }^{[3]}$. A review of the articles reporting BE (1966-2011) by Keshtkar-Jahromi et al. ${ }^{[4]}$ found that patients who did not undergo surgery had a significantly elevated risk of mortality. However, some cases of native or prosthetic BE cured with medical treatment alone have been reported ${ }^{[5-8,10]}$. Only a few cases of prosthetic valve endocarditis due to Brucella species have been treated successfully without reoperation ${ }^{[5-8]}$. In these cases, doxycycline and rifampin in combination with an aminoglycoside were used.

The antibiotic regimen and optimal duration of treatment have not yet been established. The timing of surgery is important ${ }^{[11]}$. The combination of triple antibiotic therapy for 6 weeks can be sufficient before operation ${ }^{[11]}$. After surgery, the treatment should be continued for at least 1-3 months after the Wright serological test titres have decreased below 1:160[11].

Karaoğlan et al. ${ }^{[8]}$ reported three case of Brucella mellitensis prosthetic valve endocarditis that were cured only with long-term antibiotic therapy (12 months in one patient and 6 months in the two others) until the vegetations disappeared and Brucella titres decreased to $<1: 160$. During 12 months of follow-up, patients showed no signs of relapse. Lee et al. ${ }^{[6]}$ reported the case of a 42-year-old man with a prosthetic mitral valve infected by Brucella abortus cured solely by medical therapy for 1 year, with no evidence of relapse during a follow-up period of 5 years. In our case, after 9 months of treatment, with serial TTE and serology monitoring, treatment was stopped. Two years after the end of treatment the patient remained asymptomatic and with a negative Wright test. 
We believe that the key to the successful management of this patient's BE was an accurate diagnosis, the absence of other valvular complications and no signs of congestive heart failure.

\section{REFERENCES}

1. Franco MP, Mulder M, Gilman RH, Smits HL. Human brucellosis. Lancet Infect Dis 2007;7:775-786.

2. Al Dahouk S, Nöckler K. Implications of laboratory diagnosis on brucellosis therapy. Expert Rev Anti Infect Ther 2011;9:833-845.

3. Al Dahouk S, Schneider T, Jansen A, Nöckler K, Tomaso H, Hagen RM, et al. Brucella endocarditis in prosthetic valves. Can J Cardiol 2006;22:971-974.

4. Keshtkar-Jahromi M, Razavi SM, Gholamin S, Keshtkar-Jahromi M, Hossain M, Sajadi MM. Medical versus medical and surgical treatment for brucella endocarditis. Ann Thorac Surg 2012;94:2141-2146.

5. Mert A, Kocak F, Ozaras R, Tabak F, Bilir M, Kucukuglu S, et al. The role of antibiotic treatment alone for the management of Brucella endocarditis in adults: a case report and literature review. Ann Thorac Cardiovasc Surg 2002;8:381-385.

6. Lee S-A, Kim K-H, Shin H-S, Lee H-S, Choi H-M, Kim H-K. Successful medical treatment of prosthetic mitral valve endocarditis caused by Brucella abortus. Korean Circ $J$ 2014:44:441-443.

7. Murdaca G, Colombo BM, Caiti M, Cagnati P, Massa G, Puppo F. Remission of Brucella endocarditis in a patient with mitral valve mechanical prosthesis by antibiotic therapy alone: a case report. Int J Cardiol 2007;117:e35-e36.

8. Karaoğlan I, Namiduru M, Baydar I, Yasemin ZER. Three cases of Brucella prosthetic valve endocarditis cured with medical treatment. ANKEM Derg 2009;23:46-49.

9. Lebre A, Velez J, Seixas D, Rabadão E, Oliveira J, Saraiva da Cunha J, et al. Brucellar spondylodiscitis: case series of the last 25 years. Acta Med Port 2014;27:204-210.

10. Cohen N, Golik A, Alon I, Zaidenstein R, Dishi V, Karpuch J, et al. Conservative treatment for Brucella endocarditis. Clin Cardiol 1997;20:291-294.

11. Sasmazel A, Baysal A, Fedakar A, Buğra O, Ozkokeli M, Büyükbayrak F, et al. Treatment of Brucella endocarditis: 15 years of clinical and surgical experience. Ann Thorac Surg 2010;89:1432-1436. 